Cahiers de recherches médiévales

\title{
Du Brut au Merlin
}

Le fils du diable et les incertitudes génériques

\section{Annie Combes}

\section{(2) OpenEdition}

Journals

Édition électronique

URL : https://journals.openedition.org/crm/1332

DOI : $10.4000 / \mathrm{crm} .1332$

ISSN : 1955-2424

Éditeur

Honoré Champion

Édition imprimée

Date de publication : 30 octobre 1998

Pagination : 15-32

ISSN : 1272-9752

Référence électronique

Annie Combes, «Du Brut au Merlin », Cahiers de recherches médiévales [En ligne], 5| 1998, mis en ligne le 01 octobre 2007, consulté le 15 décembre 2022. URL : http://journals.openedition.org/crm/1332 ; DOI : https://doi.org/10.4000/crm.1332

Ce document a été généré automatiquement le 15 décembre 2022.

Tous droits réservés 


\title{
Du Brut au Merlin
}

\author{
Le fils du diable et les incertitudes génériques
}

\section{Annie Combes}

Écrire en prose, au début du XIII ${ }^{\mathrm{e}}$ siècle, c'est une voie que choisit un auteur qui déclare se nommer Robert de Boron, et c'est un choix dont il est difficile d'appréhender les raisons. Nul commentaire métadiscursif, nul aveu auctorial et, non plus, nul traité théorique ne nous éclairent sur la nécessité qui a poussé un écrivain à transcrire en prose un texte qui existait déjà en vers, le Joseph d'Arimathie, sans en modifier de façon notable le contenu ${ }^{1}$. La naissance du récit en prose ne relève donc pas de la rupture, mais d'un progressif démarquage ${ }^{2}$. Toutefois, ce n'est pas au Joseph que je vais m'intéresser, mais à celui qui constitue sa «suite», le Merlin en prose ${ }^{3}$. Là encore, on constate le phénomène de réduplication vers/prose, mais de façon restreinte car subsistent dans un unique manuscrit cinq cents vers d'un Merlin octosyllabique, sans que l'on puisse dire si ce vestige est tronqué ou si a pu exister un Merlin en vers, complet $^{4}$. La tradition manuscrite est bien plus généreuse pour la prose, puisque de nombreux témoins présentent l'enchaînement Joseph-Merlin; deux d'entre eux leur ajoutent un Perceval (où l'on voit in fine mourir la chevalerie arthurienne), formant ainsi ce que l'on appelle le cycle du Petit Saint Graal'.

2 Si l'on considère ces trois œuvres, on est frappé par leur diversité sémantique : à la pieuse histoire de Joseph, contemporain exemplaire du Christ, succède l'évocation souvent facétieuse de la vie de Merlin, le fils d'un diable, tandis que le dernier ouvrage met en scène les aventures très romanesques de l'intrépide Perceval, jusqu'à la chute du royaume arthurien. J'estimerais volontiers que si ces textes, bien qu'en continuité au niveau de la diégèse, sont de thématique aussi disparate, c'est parce qu'il n'existe pas en ce qui les concerne de base pour leur homogénéisation. Ils n'ont aucune identité générique évidente et l'on peut penser que leurs conditions d'émission et de réception n'avaient pas, au moment de leur production, d'ancrage pragmatique, contrairement par exemple à la chanson de geste, dont le rôle social était bien marqué. Le choix d'une même forme - la prose - ne s'est accompagné d'aucune sélection très nette de la matière, si bien que la suite de textes reste sémantiquement hétérogène, et que le Merlin, au centre de la série, présente des caractéristiques thématiques et stylistiques 
qui lui sont propres. Ce que je souhaiterais faire dans ces pages, c'est tenter de cerner son identité générique, poser quelques jalons pour comprendre la nature et les ambitions de ce texte, l'un des premiers qui ait assumé la forme prose.

Cet objectif sera envisagé par la mise en perspective du Merlin avec une œuvre qui en constitue une source importante, le Brut de Wace, un récit en vers qui a fourni à l'œuvre en prose son héros éponyme, les circonstances de sa naissance et la nature du rôle tenu par le prophète auprès des rois bretons Uter et Arthur ${ }^{6}$.

4 En s'engageant sur une telle piste, il ne faut pas dissimuler le risque que l'on prend à vouloir parler de spécificité générique pour une époque qui ne pense pas la littérature en de tels termes, ne raisonne pas de la sorte. L'auteur du Merlin ne semble pas avoir eu de modèle textuel en tête lorsqu'il a composé son récit - et l'on va voir combien en effet les traits qui caractérisent son ouvrage sont disparates. Par conséquent, attribuer à ce livre l'étiquette de roman, comme on le fait souvent, occulte ce qu'il y a de problématique dans sa nature. Davantage, une telle démarche masque la difficulté qu'il $\mathrm{y}$ a à classer ce texte. Ou, pour le dire autrement, le Merlin ne fut pas écrit en fonction d'un genre préalable dans lequel son auteur aurait voulu l'inscrire, mais au contraire, il manifeste un état de créativité relativement libre: de même que son personnage éponyme affirme "je sui oscurs " ${ }^{7}$, le livre qui le contient est d'identité obscure dans la mesure où il oscille entre plusieurs aspirations, et se réalise à travers des thématiques variées. La recherche, non pas d'un "genre ", mais de "traits génériques " paraît donc ici la seule envisageable, voire la seule légitime. Ces traits génériques seront entendus comme des composantes énonciatives et sémantiques, dont l'association éventuellement, aussi, la hiérarchie - construit la physionomie de l'œuvre, et non comme des indices marquant une appartenance à un hypothétique genre littéraire préexistant $^{8}$. En comparant les traits génériques du Merlin et du Brut, ce sont aussi les choix esthétiques de la prose qui se manifesteront par rapport au modèle versifié.

J'examinerai d'abord les aspects qui caractérisent la généricité fuyante du Merlin, puis je me tournerai vers le Brut pour y découvrir une organisation plus nette. Enfin, à partir de la confrontation de ces deux ouvrages, je tenterai de dégager les caractéristiques de l'œuvre en prose, dont on verra qu'elles ne correspondent pas exactement aux idées habituelles que l'on s'en fait.

Une pluralité d'écritures

6 Le cadre communicationnel joue un rôle très important dans le Merlin, c'est lui qui fonde en grande partie la diversité sémantique du récit ${ }^{9}$. Dans le cours de l'œuvre, on voit surgir par deux fois le nom d'un énonciateur d'allure réelle, mes sires Roberz de Borron, un nom d'auteur ${ }^{10}$ (c'est ce nom que l'on trouve déjà dans le cours des Joseph en vers et en prose), que relaie un «je», les deux venant s'ajuster lisiblement l'un à l'autre à la fin de l'ouvrage, avec les mots «Et je Rebert de Borron... ». Ce «je » s'adresse à un destinataire indéterminé, marqué simplement par des adresses: «En cel contemple dont je vos ai parlé... » ou «si com je vos ai conté », ou " cil [Blaise] les mist en escrit et par lui le savons nos encore $»^{11}$. Le destinataire est un être plutôt collectif, un auditeurlecteur, dont la réalité et celle de Robert se renforcent mutuellement.

7 Les interventions nombreuses $\mathrm{du}$ « je » mettent le plus souvent en récit la production de l'œuvre et reconnaissent la dette de Robert à l'égard d'un livre dont il dit incessamment s'inspirer. Cette procédure de délégation de l'origine serait banale si le livre en question n'était, aussi, celui dont le récit lui-même raconte l'élaboration. Comme on 
sait, l'histoire du Merlin, c'est l'histoire de l'écriture d'un livre dicté par Merlin, dont le titre, mentionné dans le cours de l'œuvre par le prophète lui-même, est le Livre dou Graal : la diégèse montre donc l'élaboration d'un ouvrage qui n'est pas celui que l'on est en train de lire, mais qui en est la source. D'où un phénomène de démultiplication dans l'identité de l'ouvrage, en relation avec la présence de ces deux énonciateurs. Même si l'un, Robert de Boron, est censé surplomber l'autre, la hiérarchie des instances énonciatrices n'est pas toujours claire. Entre le livre de Blaise et celui de Robert, les différences sont difficiles à repérer, puisque nous n'avons accès au «biau livre» dicté par Merlin qu'à travers ce que nous en dit Robert! L'écart, d'ailleurs, tend à être minimisé par l'énonciateur hétérodiégétique, lorsqu'il note que Merlin, après l'établissement de la Table Ronde, vient voir Blaise et «li dist ces choses et les establissemenz de ceste table et maintes autres choses que vos orroiz en son livre ${ }^{12}$, confondant alors l'ouvrage dicté par Merlin et le sien propre.

On constate pourtant une tentative de distinction entre les activités respectives des deux énonciateurs: Merlin «aconte les amors de Jhesu Crist et de Joseph", il «dita ceste oevre et la fist faire a Blaise ", il «conta ces choses", ou encore il part rejoindre Blaise en Northumberland «por raconter toutes ces choses et autres choses por baillier matiere a son livre faire $»^{13}$. Le résultat du travail de Blaise, lorsqu'il a usé encre et parchemin, c'est un livre, dans lequel est inscrite l'œuvre de Merlin. Quant à Robert de Boron, il «retrait cest conte»; occasionnellement, il précise qu'il y a certaines choses qu'il ne peut ou ne doit «retraire $»^{14}$. Le mot qui revient pour désigner l'œuvre qu'il élabore, c'est « conte », souvent précédé du démonstratif « cist/cest $»^{15}$.

9 L'examen des termes employés par les préposés à la dictée/écriture fait ainsi apparaître une frontière interne au texte entre «livre» et «conte». Ce que le lecteur découvre, c'est le « conte » que Robert « retrait »; Merlin, lui, raconte et dicte après avoir vécu. Le relevé manifeste l'imbrication de l'écrit et de la diction, dans l'imaginaire de la production de l'œuvre.

10 L'ouvrage auquel nous, lecteurs, avons accès, est donc de nature hybride. C'est, et ce n'est pas, le livre de Merlin. Robert de Boron raconte le contenu de ce livre, mais l'ouvrage qu'il produit se différencie de son modèle sur au moins deux aspects. Le premier, bien sûr, c'est la présence de Robert: messire de Boron n'appartient pas au livre de Merlin, dont il n'est que le lecteur! Le deuxième, c'est l'influence perceptible d'un autre livre dont la matière paraît exogène au Livre dou Graal, car elle est constituée de scènes guerrières dont on ne voit pas pourquoi le prophète les aurait mises en mémoire, et dont rien n'indique qu'il l'ait fait. Ainsi, lorsqu'à propos de la bataille de Salesbières, Robert de Boron précise que «li livres raconte que Uitiers veinqui la bataille $»^{16}$, c'est peut-être bien le Brut que désigne ici le mot «livre »... Ce lapsus est d'autant plus plausible que Merlin, qui est resté en dehors de l'événement, ne se précipite pas vers Blaise pour lui « dire ces choses».

11 Le Merlin présente en somme des effets de décalage entre la revendication testimoniale d'un énonciateur qui garantit la fiabilité de son propos par sa propre fidélité au livre qu'il «retrait» et la capture de la diégèse par un énonciateur tel que Merlin. Ce dédoublement remet en cause le crédit que l'on peut faire à l'ensemble, en révélant qu'il s'agit d'une pseudo-histoire. Cette ambiguïté est corroborée par la diversité d'une thématique qui hésite entre le miraculeux d'une part (qui relie le Merlin, en amont, au 
Joseph), le merveilleux et l'historique d'autre part (qui tous deux se retrouveront dans le Perceval).

L'approche thématique est délicate : le repérage des traits de contenu ne va pas de soi car, on l'a dit, le sujet du Merlin ne s'impose pas à l'analyse. Le récit s'ouvre sur un temps postérieur à la mort du Christ, dans un lieu extraordinaire, en Enfer. Le conseil des diables que le récit met en scène sous forme dialoguée ${ }^{17}$ se clôt sur la décision de faire naître un Antéchrist capable de repeupler les espaces infernaux devenus déserts depuis la Rédemption. Suit une évocation des ravages effectués par un diable au sein d'une famille, jusqu'à la conception de Merlin. Viennent alors l'évocation de la grossesse de la mère et, après l'accouchement, les premiers prodiges de l'enfant. Au juge qui veut condamner sa mère à mort, Merlin explique les particularités de sa naissance et comment les diables ont échoué dans leur projet. Il prouve en outre que l'homme respectable qui le juge est un bâtard. Ensuite, il demande au prêtre Blaise d'écrire le livre que lui, Merlin, dictera. Le roi breton Vertigier fait alors son entrée dans le récit: sur le conseil de ses clercs, il envoie des messagers à la recherche d'un fils sans père. Leur mission les conduit en Orient, d'où ils ramèneront Merlin. Durant le voyage vers l'Occident, deux scènes («le vilain qui achète du cuir», «l'enfant du prêtre ») confirment les talents prophétiques de l'enfant. Par la suite, Merlin donne quelques conseils à Vertigier, puis devient l'auxiliaire militaire de ses successeurs, Pendragon et Uter, qu'il charme par ses plaisantes métamorphoses. A l'occasion, il interprète l'apparition de dragons terrestres ou célestes. Et c'est lui qui aide Uter à séduire Ygerne, en changeant l'apparence physique du roi, provoquant ainsi la naissance d'Arthur. De façon de plus en plus appuyée, le récit se concentre sur les rois de la Grande Bretagne, jusqu'à ce qu'Arthur lui-même accède au trône.

La variété sémantique est flagrante. Surtout si l'on songe au Joseph, ce récit constant dans le ton, l'esprit et le propos qui, adoptant le point de vue de Joseph d'Arimathie, présente la propre «mise au tombeau » de ce personnage exemplaire et l'intercession divine lui permettant de rester en vie ; puis, après sa « résurrection ", le sort de la petite communauté pieuse qui se groupe autour de lui. A cette œuvre pourrait être attribué un trait générique "scripturaire", puisqu'elle raconte, à la manière d'un évangile, la Passion du Christ et ses suites. Ce trait scripturaire s'illustrerait en outre par un trait «miraculeux », en relation avec les prodiges accomplis par le Christ ou par son précieux auxiliaire, le Graal.

Le Merlin n'a plus la même unité de ton ni de thème, même si le récit trouve son centre génétique dans son héros éponyme, dont est racontée la vie depuis sa naissance jusqu'à son rôle de conseiller auprès des souverains bretons. Mais cette créature polymorphe, ce fils d'un diable et d'une pieuse femme, fait basculer l'écrit dans un alliage générique insolite.

L'œuvre présente en fait une composition en deux parties, avec comme pivot l'endroit où il est fait pour la première fois allusion à Vertigier. Tout ce qui précède peut être perçu comme un vaste prologue, dans lequel on retrouve les traits génériques du Joseph, le «scripturaire " associé au "miraculeux». Toutefois, ces lignes de crête sémantiques ne s'imposent pas aussi fortement car leur domaine d'application est réduit. En outre, la dimension apologétique n'est pas nette, contrairement au Joseph. Quelques phrases, seulement, qui ponctuent les malheurs de la famille décimée par le diable, prouvent une intention édifiante, telles celles-ci : 
Et einsi poez savoir que molt est deables fols, et molt devons estre irié, quant si fole chose nous engingne. [...] Et einsis fait deables de cels qu'il puet engingnier et que il treuve a sa volenté. [...] Einsis jeue deables de cels qui consentent sa volenté en terre. ${ }^{18}$

L'énonciateur intervient même pour renforcer la visée moralisatrice. Mais ensuite prennent place les scènes du procès, qui permettent à Merlin de ridiculiser le juge et ajoutent un trait satirique au faisceau qui se constitue.

Dans la seconde partie, une composante historicisante s'impose, qui se maintient jusqu'à la fin de l'œuvre. Elle voisine avec le trait «merveilleux », qui prend le relais du trait «miraculeux». Des merveilles surgissent en effet occasionnellement : apparition de dragons ou d'une pierre flottante dans laquelle est fichée l'épée réservée à Arthur, transfert de Stonehenge d'Irlande en Grande Bretagne, effets terrifiants du «siège vide» de la Table Ronde ${ }^{19}$. Le trait "satirique", quant à lui, a laissé place, dans un registre proche, à un trait « comique», apparent lorsque Merlin multiplie les tours pour amuser les rois. La composante «scripturaire » a disparu, à moins que l'on ne considère qu'elle s'est réfugiée dans le thème récurrent de l'écriture de Blaise.

18 En somme, on découvre une œuvre qui prend par endroits des allures de récit de miracle, ailleurs de fabliau, ailleurs encore tend vers l'historiographie, qui juxtapose le plan céleste avec les choses humaines vues par Merlin. À partir de l'évocation de Vertigier, le récit est marqué par un trait indubitable d'historicité, mais qui n'englobe pas tous les autres traits. Leur mode d'existence est davantage la juxtaposition que l'inclusion. L'impression d'hybridité générique s'atténue sans doute, sans toutefois disparaître. Bref, le Merlin présente un ensemble de traits sémantiques qui s'ajoutent davantage qu'ils ne se mêlent, formant un spectre dont les composantes se modifient à mesure : on pourrait parler de généricité évolutive.

Incertitude sur le statut de l'énonciateur, variété et instabilité des traits génériques: tout cela met en question l'identité véridico-historique du récit. On retrouve cette indécision dans la mise en œuvre d'une temporalité ambigüe, dont le statut va évoluer au cours de l'ouvrage: l'organisation temporelle confirme l'hybridation des composantes génériques.

20 En effet, à l'ouverture du récit, Merlin évolue dans une époque chrétienne indéfinie, dont on peut seulement comprendre qu'elle se déploie à partir de la Passion du Christ. Le Merlin faisant suite au Joseph, on pourrait s'attendre à une continuité entre les deux ouvrages, le second débutant au moment où le premier s'achève, lorsque les gardiens du Graal partent vers l'occident. Mais les événements relatés dans le Joseph ne couvrent que quelques décennies (moins d'une vie humaine, puisque Joseph est adulte au début du récit et qu'il meurt à la fin), bien insuffisantes pour parvenir à l'époque d'Arthur. En centrant son récit sur Merlin, Robert de Boron fait un saut dans le temps, mais il masque l'hiatus en situant sa diégèse dans un lieu extraordinaire, l'enfer, et en mettant en scène des diables effondrés par le vide qui règne dans leurs demeures depuis que le Christ a racheté les péchés des hommes ${ }^{20}$. L'analepse, parce qu'elle est de portée indéterminée, masque la rupture temporelle, crée même un effet de correspondance avec le début du Joseph dans la mesure où elle se situe dans le registre chrétien.

21 Ce temps imprécis finit cependant par prendre une forme plus concrète. Cela se produit à l'endroit charnière déjà mentionné, lorsque l'énonciateur intervient pour situer la diégèse dans le sol et surtout dans l'histoire objective de l'Angleterre. Alors qu'il a été 
longuement question de l'élaboration du livre de Blaise et après une phrase où Robert de Boron se nomme pour la première fois, surgit cette assertion :

En cel contemple dont je vos ai parlé et dont je vos parole encore estoit novelement venue crestientez en Engleterre, n'il n'i avoit encore gaires rois crestiens euz. ${ }^{21}$

La rupture est fortement visible, même si le terme «crestientez » assure la liaison avec la thématique religieuse qui baignait la première partie du récit. Mais avec cette phrase, on va entrer dans une temporalité plus laïque. L'introduction de ce temps historique est indispensable dans l'économie de l'œuvre pour amener Merlin d'Orient en Occident, et pour rejoindre l'époque arthurienne. Comme l'écrit Alexandre Micha, «il fallait mettre le lecteur au courant de la conjoncture historique, introduire Vertigier dans l'action, puisque le chemin de sa destinée va se croiser avec celui de Merlin $»^{22}$. Le récit, qui se développait jusqu'alors sans repère précis, épouse dès lors ce déroulement chronologique dont le modèle lui est fourni, bien sûr, par le Brut. L'agencement est fort habile. Succédant de la sorte à la Passion et à la Rédemption, le temps des rois bretons reçoit une aura spirituelle, et, en ce sens, l'organisation du Merlin dévoile le projet central de l'œuvre, et même celui du cycle : associer la matière de Bretagne à l'essor d'une chrétienté magnifiée et fortifiée par le Graal.

Au cœur du dispositif: Merlin, ou plus exactement la vie de Merlin, qui permet l'écriture du livre de Blaise, et par là-même l'activité littéraire de Robert de Boron ${ }^{23}$. En insistant sans cesse sur le rôle du prophète comme source du livre, le Merlin met en avant une origine fictive, qui occulte l'origine effective de la fiction, laquelle se trouve dans le Brut. En somme, la tradition livresque réelle, qui part de l'Historia Regum Britanniae et passe par le Brut pour aboutir au Merlin est transmutée en une filiation légendaire qui repose sur le livre de Blaise. C'est pourquoi il importe d'examiner à présent la nature générique de ce modèle intertextuel éludé par le Merlin, afin de mieux définir son rapport avec le récit en prose de Robert de Boron.

Une histoire de rois

L'ouvrage de Wace est une œuvre en vers octosyllabiques qui raconte l'histoire des Bretons. Wace s'attache à traduire l'Historia Regum Britanniae de Geoffroy de Monmouth, écrite en latin ${ }^{24}$. Dans une étude devenue classique, Robert Marichal parle du Brut comme d'un "roman historique $»^{25}$. Le terme de "roman" paraît d'un emploi bien téméraire ; comme on va le voir, celui de « chronique » le serait certainement moins.

Le récit est pris en charge par "Maistre Wace», nommé dans la première phrase de l'ouvrage. Cette troisième personne laisse place bientôt à la première, à l'occasion de l'assertion suivante, à propos des dieux troyens qui ne vinrent pas à Albe : «El temple ralouent ariere, / Mais jo ne sai en quel maniere $»^{26}$. Wace l'auteur intervient donc à l'occasion dans son récit en usant du «je», dans des assertions qui précisent ses capacités cognitives. Cette aptitude interprétative s'exerce principalement sur l'ouvrage que Wace «translate », avec un souci de fidélité qu'affiche par exemple le vers $9:$ «Si cum li livres le devise ». On reconnait là encore le procédé du livre originel, mais cette fois-ci la source est réelle. Dans le cours du récit, Wace ne manque pas de rappeler fréquemment son statut premier de lecteur ${ }^{27}$, et son statut de traducteur. On rencontre ainsi deux fois le mot « romanz » qui, conformément à son étymologie, réfère à l'activité de traduction qui sous-tend la production du texte: «Ki cest romanz fist, maistre Wace » et « Fist mestre Wace cest romanz $»^{28}$.

Mais le mot « livre» se rencontre aussi lorsque Wace évoque son propre ouvrage : 
Maistre Wace, ki fist cest livre,

Ne volt plus dire de sa fin

Qu'en dist li prophetes Merlin ;29

la nature de l'œuvre, aussi bien sur l'original que la transposition. On trouve ainsi employés, au moment de l'évocation de la grande fête à la cour du roi Arthur, les mots «estorie de la geste», qui s'appliquent à la source de Wace, c'est-à-dire au livre de Geoffroy de Monmouth:

Al matin, le jur de la feste, Ço dit l'estorie de la geste,

Vindrent tut trei le arcevesque [...]. ${ }^{30}$

29 À la fin de son œuvre, Wace annonce "Ci falt la geste des Bretuns ${ }^{31}$. Ces termes d'« estorie » et de "geste» situent nettement le propos sur le plan de l'historiographie ; les res gestae, on le sait, pouvaient désigner le récit résumant les actes officiels des souverains, et la thématique globale du Brut se situe bien dans cette optique. Wace déclare faire une "estorie", et, même s'il reste délicat de parler dans son cas de chronique ou d'histoire proprement dite, je pense qu'il faut placer au premier rang le trait d'« historicité » pour comprendre son projet. On voit alors que ce trait essentiel est illustré par un ensemble de traits convergents, de nature formelle et sémantique.

Tout d'abord, le choix de l'octosyllabe doit se comprendre par référence à l'une des rares œuvres qui ait employé le couplet octosyllabique avant Wace : l'Estoire des Engleis, de Geffrei Gaimar ${ }^{32}$. Cet auteur s'est lui-même fondé essentiellement sur une annale anglo-saxonne, tout en compilant d'autres sources. Wace reprend ce modèle métrique et comme Gaimar encore, il adopte une organisation linéaire qui mime la continuité des règnes. Le projet est de faire savoir au lecteur «quels reis $i$ ad en ordre eü $»^{33}$. Wace transpose donc un livre d'Historia (de Geoffroy) en adoptant la forme versifiée utilisée par Gaimar pour sa propre Estoire. Il emprunte à l'un sa matière (qu'il complète par des connaissances acquises grâce à la lecture d'autres ouvrages, comme a pu l'établir son éditeur), à l'autre sa forme métrique, dans l'unique projet de faire œuvre historiographique.

31 La structuration par successivité qu'il adopte s'accompagne d'un tri des événements liés aux rois et aux terres conquises, partagées, perdues, retrouvées... Wace s'attache tout particulièrement à marquer et dérouler la continuité. Il a donc recours à des précisions qui, en mentionnant l'écoulement de la durée, évitent de laisser des temps vides: d'où l'emploi de termes tels que «lungement », «emprés lung tens », et les vers tels que «Quarante anz fu reis, puis fina $»^{34}$.

Enfin, ces traits formels de l'historicité sont complétés par deux sous-traits sémantiques, "didactisme» et "véridicité», qui viennent épauler le projet historiographique.

L'ambition didactique est affirmée dès le court prologue, et on la retrouve dans le cours du récit, dont elle modèle la matière: Wace décrit les lignages des rois. En outre, comme Geoffroy de Monmouth avant lui, il s'appuie sur le temps référentialisé qui a 
laissé des traces dans la toponymie. Le Brut est en effet émaillé d'explications étiologiques sur les noms des villes, racontant l'histoire de leur dénomination, depuis une époque parfois lointaine où tel personnage, tel événement imprima sa mémoire dans la toponymie. Ainsi de Tours :

Pur Turno qu'illuec fu ocis

E el chastel en terre mis

Fu puis Turs la vile apelee

E Toroinne entur la contree. ${ }^{35}$ l'Eneas ou Cligès, fondée sur la répétition, l'énumération et l'antithèse. Voici en effet comment s'exprime Uter :

L'amur d'Ygerne m'ad suspris,

Tut m'ad vencu, tut m'ad conquis,

Ne puis aler, ne puis venir,

Ne puis veillier, ne puis dormir,

Ne puis lever, ne puis culchier,

$\mathrm{Ne}$ puis beivre, ne puis mangier,

Que d'Ygerne ne me suvienge ;0 
39 Jerai volontiers d'un trait "pré-romanesque ", qui échappe au projet principal de l'œuvre. Comme il demeure isolé, il ne suffit pas à contester pas le projet historiographique. Il amène plutôt une variation de tonalité.

Sur un plan tout différent, le long descriptif du combat qui oppose Goemagog et Corineüs semble également s'éloigner du cadre de la chronique pour se rapprocher d'autres formes narratives où l'on voit ainsi exaltées la force et la combativité des héros ${ }^{41}$. Le style de Wace prend alors une vigueur inhabituelle grâce à des effets d'anaphore et de répétition et par des adresses au lecteur telles que «Dunc veïssez » que l'on retrouve ailleurs dans le récit lorsqu'il s'agit d'évoquer une bataille :

\footnotetext{
Braz a braz se sunt entrepris,

Bras unt desus e desuz mis.

Es les vus ensemble joztez,

Piz contre piz, lez contre lez;

Par detriés les dos s'enbracerent,

Par grant aïr lur mains lacerent ;

Dunc veïssez tur contre tur,

Vigur mettre contre vigur,

E pied avant e pied ariere,

E engieng de mainte maniere $;^{42}$
}

41 La durée de la description et les reprises lexicales produisent un écart vis-à-vis du projet strictement historiographique, mais, pour autant, aucun trait précis ne surgit. En particulier, le rythme sautillant de l'octosyllabe, particulièrement sensible dans ces vers, donne au combat l'allure d'une danse concertée bien éloignée de la tonalité épique. Simplement, le spectacle des combattants est extrêmement détaillé, et l'image de leur agilité physique donne à voir la virtuosité verbale de l'auteur ${ }^{43}$.

Limitée ainsi à de brèves unités narratives, c'est davantage une diversité stylistique qui se manifeste que l'affirmation de traits génériques nouveaux. Ces tendances demeurent secondaires parce qu'elles n'engagent que de courts passages de l'œuvre, et qu'elles figurent dans des scènes à la fois isolées et disparates. Mais peut-être plus troublants sont les passages où sont évoqués des phénomènes que nous rangerions sans hésitation dans le fabuleux, tel le développement sur les sirènes, ces créatures mentionnées en une seule phrase dans l'Historia Regum Britanniae, et qui sont décrites en trente vers par Wace ${ }^{44}$. Ces «monstres de mer» qui «duces voiz unt, dulcement chantent» mettent en péril les marins: les vers s'attardent avec délectation sur ces créatures qui «figure portent de Diable», tandis que, sur le plan de l'action, rien n'advient, car les Troyens ne les écoutent pas.

On pourrait considérer qu'il y a là un glissement de l'historia vers la fabula, que l'on a un trait générique qui pointe vers la légende, dans ce qu'elle a de merveilleux. Mais il n'est absolument pas sûr que les sirènes aient été perçues par Wace comme des créatures chimériques; il semble plutôt situer ces êtres étranges dans la sphère culturelle chrétienne, neutralisant de la sorte toute dimension imaginaire, comme il le fait également avec la «devineresse» Diane, dont il dit que «Diables esteit, ki la gent/ Deceveit par enchantement " ${ }^{45}$. Le mot «diable " constitue une façon de ramener dans la sphère du vrai ce que l'on pourrait suspecter d'être purement légendaire.

Et Merlin? Effectivement, avec le fils du diable, le propos de Wace atteint l'une de ses limites, mais il repousse le plus longtemps possible le surgissement de la fabula : ce n'est qu'avant de disparaître de la diégèse que Merlin manifeste sa nature de magicien, en 
changeant son apparence et celle d'Uter et d'Ulfin (je vais y revenir). Mais jusque là, le Brut présente les caractéristiques génériques suivantes : une forme versifiée continue, un trait essentiel d' "historicité», avec des sous-traits de «didactisme» et de "véridicité». Hormis le passage où l'on voit agir l'enchanteur, cette structuration générique s'applique, me semble-t-il, à la globalité de l'ouvrage.

On est à même maintenant de définir le grand changement sémantique qui se produit entre le récit de Wace et le Merlin : aux réticences de Wace par rapport au prophète va s'opposer le développement fictionnel que Robert de Boron accorde au personnage. On comprend que les définitions génériques des deux ouvrages soient aussi différentes, alors même que l'un s'inspire directement de l'autre.

Essayons d'appréhender les raisons de ce bouleversement des traits génériques.

Un modèle escamoté

Dans l'ouvrage en prose est effectuée une sélection de la matière, puisqu'une partie seulement de l'écrit de Wace y est reprise. Robert de Boron emprunte le personnage de Merlin et réutilise des données de sa biographie de façon assez fidèle, même si sa transposition ne respecte pas toujours l'ordre exact des événements ${ }^{46}$. L'apparition du trait d'historicité se produit lorsque le récit capte précisément la temporalité du Brut pour en faire son axe directeur, alors que jusque-là, la diégèse évolue dans un temps imprécis mais de tonalité chrétienne. La clôture de l'œuvre correspond à la fin de l'épisode consacré au prophète dans le Brut.

Pourtant, malgré ces coïncidences globales, un renversement s'opère dans la place attribuée au trait d'historicité : premier et permanent chez Wace, il n'est présent que dans un second temps chez Robert de Boron. En outre, lorsqu'il se manifeste dans la prose, c'est pour voisiner avec d'autres traits, avec lesquels il semble en concurrence. Deux explications peuvent être avancées; l'une concerne la nature des temps mis en jeu, l'autre repose sur des phénomènes d'amplification et de réduction, manifestes dans le Merlin.

Le Merlin se fonde sur un temps chrétien et non historique, qui n'apparaît qu'en pointillés dans le Brut. En effet, chez Wace, le temps chrétien se déroule en marge du temps de l'Histoire, comme c'est aussi le cas chez Geoffroy de Monmouth. Interrompant le récit, une précision indique qu'«A cel terme que jeo vus di $»^{47}$, Hélie était prêtre de Judée, Saül était roi des Juifs, Jésus Christ naissait ${ }^{48}$... Ce temps annexe du Brut devient le temps originel du Merlin, qu'il faut quitter pour entrer dans l'histoire. C'est alors seulement qu'advient le saut temporel, au moment où l'énonciateur mentionne abruptement la présence de rois chrétiens en Angleterre.

Par ailleurs ce temps historique est représenté dans la prose en accéléré, traité comme une nécessité, sans développements. Dès son surgissement, on a le sentiment d'un résumé obligé, introduit pour parvenir à mettre en contact Merlin et Vertigier. Cette caractéristisque va être constante: la pratique de l'abrègement s'applique sur tous les passages du Brut où il est question des combats contre les Saxons ; l'une des campagnes contre Octa est même omise ${ }^{49}$. L'énonciateur de la prose précise bien les limites de sa tâche: «Des rois qui i avoient esté ne me tient riens a retraire » ou encore «Tant fist Engis et tant porchaça maintes choses que je ne vos doi retraire... $"^{50}$.

51 Les passages marqués par le trait d'historicité sont ainsi raccourcis et séparés par des scènes dont j'ai déjà signalé les caractères très différents du point de vue générique. Mais il convient de voir que les autres traits qui apparaissent alors dépendent tous du 
personnage de Merlin, dont la présence est rendue plus massive par l'amplification que subissent plusieurs passages du Brut, en particulier l'épisode de la tour de Vertigier, le retour des messagers jusqu'en Angleterre, l'explication des dragons, le transfert de la «carole des géants» et les métamorphoses de Merlin, Uter et Ulfin. Dans la prose, les dons du prophète et ses relations avec les rois bretons prennent ainsi une ampleur nouvelle. Comme le dit Paul Zumthor, avant le livre de Robert de Boron, Merlin « n'était qu'un nom, un message, une voix sans personnalité »; grâce à Robert (et au livre de Blaise !), « il s'est incarné »15.

Si Robert de Boron retire une part de son rôle à l'historicité, il conserve une caractéristique qui en découle, la continuité. De ce point de vue, l'idée que la prose est davantage liée au temps que les vers apparaît comme une idée préconçue. Le Brut offre en effet l'exemple d'une remarquable traversée temporelle, avec des principes clairs et efficaces. Robert, à son tour et à l'intérieur des limites temporelles de son propre récit, s'évertue à recréer cet effet de chronologie dont on a vu comment il était soigneusement produit dans le Brut. Si l'on examine la construction du Merlin, on constate qu'il est formé d'une succession de situations présentant, chaque fois, une unité temporelle, et associées par continuité, sauf au moment où est introduit Vertigier ${ }^{52}$. Le conseil des diables ouvre directement sur les actes de l'incube, qui favorisent la conception de Merlin; alors débute un temps vide - celui de la grossesse de la mère - à l'occasion duquel apparaît pour la première fois la formule que Robert de Boron utilisera ensuite avec prédilection pour marquer la continuité et donner l'idée d'un déroulement : «Einsis remest... $»^{53}$. L'emploi de ce verbe est récurrent, à côté des procédés syntaxiques bien connus et que l'on trouve déjà dans le Joseph: les subordonnées en «tant que», ou l'enchaînement «quant... si ». Voici quelques phrases où ces procédés sont bien représentés, lorsque Robert de Boron résume les événements historiques qui précèdent l'accession de Vertigier au trône :

Et li Saine se combatirent a els, si les veinquirent et desconfirent; et quant il furent veincu et desconfit, si s'en revinrent et distrent que molt avoient perdu et qu'il n'eussent pas ceste perte faite, se Vertigiers i eust esté. Einsi remest. Li enfes ne savoit mie si bien les genz retenir ne avoir com mestiers li fust: si li cuillirent li plusor en haine. Einsi remest une grant piece, tant que cil fu si tenuz a mauvais qu'il distrent qu'il ne sofferroient plus. ${ }^{54}$

53 L'adverbe « einsi » rythme la prose du Merlin, marquant tous ces moments où le temps s'écoule sans que rien ne change. L'auteur du Merlin doit au Brut non seulement le personnage du prophète mais la notion du dévidement temporel, une ambition que les romans en vers de la seconde moitié du XII siècle ne manifestent pas : c'est seulement avec la prose, et plus particulièrement avec le Merlin, que réapparaît un intérêt pour la chronologie, mais celui-ci, comme on le constate, trouve son modèle dans une chronique versifiée.

Le trait générique d'historicité, qui n'est plus dominant, subsiste donc à travers le souci de la chronologie, de la durée. Mais il est atteint encore à travers le sort qui est fait aux deux sous-traits qui l'accompagnent dans le Brut. Le didactisme ne survit qu'à travers la composante moralisatrice; quant à la véridicité, elle est sérieusement mise à mal. On remarque en effet dans la prose la suppression de tout ce qui, chez Wace, tend à rationaliser la part de fabula qui environne Merlin.

Wace a fort à faire pour préserver le sérieux de son entreprise lorsqu'il relate la naissance de Merlin, qu'il met en scène les dragons volants, qu'il explique le transfert 
des pierres de Stonehenge d'Irlande en Cornouailles, ou enfin qu'il décrit les métamorphoses d'Uter, d'Ulfin et de Merlin lui-même. Pour montrer les différences de stratégie suivies par Wace et Robert de Boron, je vais revenir brièvement sur chacune de ces scènes.

Dans le Brut, la mère de Merlin raconte comment un être invisible, mais qu'elle pouvait entendre et toucher, lui rendait souvent visite. Et un jour, dit-elle, «Od mei se culcha, si conçui »55. Vertigier fait venir « un clerc de lettres mult savant» et lui demande si cela est possible. Le clerc fournit alors une explication circonstanciée, dont je ne retiendrai que les quatre vers suivants:

Trové avum, dist il, escrit,

Qu'une manere d'esperit

Est entre la lune e la terre. [...]

Incubi demones unt nun $; 56$

Le caractère invraisemblable de la conception est donc envisagé intradiégétiquement, et c'est à ce niveau-là qu'il est réglé, sans que soit nécessaire l'intervention de l'énonciateur. La naissance de Merlin se trouve authentifiée par le recours au texte écrit, dans une démarche calquée sur celle de Wace lui-même, qui note à l'occasion, par exemple au vers 946 , «si come je la truis escrite $»^{57}$. L'emploi de mots latins pour nommer le phénomène va dans le même sens, celui du plausible, afin de ne pas mettre en péril la fiabilité de l'œuvre. Ce souci disparaît dans le Merlin, où l'épisode est longuement développé, où l'affaire de l'incube est montrée dans tout son déroulement au lieu d'être résumée par la mère. Cette amplification amène des traits de moralisation, et le caractère prodigieux de l'affaire n'est pas gommé, parce que, on l'a vu, il s'inscrit dans la sphère du miraculeux chrétien (auquel ressortit le diabolique) que le Joseph a déjà illustrée.

On comprend que pourrait se créer dans le Merlin une tension dialectique entre l'historia et la fabula. L'œuvre conserve juste ce qu'il faut d'historicité pour étayer un temps objectif. Mais le merveilleux (ou sa variante miraculeuse) se déploie là même où, dans le Brut, il est circonscrit, rationalisé le plus possible, et entouré de larges développements guerriers à caractère historique. Avec Robert de Boron se déploient les merveilles, comme l'atteste déjà sur le plan du vocabulaire l'emploi fréquent de ce mot.

Une autre illustration de ce changement de perspective est fournie par le dragoncomète du Brut. Pour Wace, comme pour Geoffroy de Monmouth, au moment de la mort d'Aurèle, une « esteille » apparaît, sur laquelle sont données les précisions suivantes :

Cumete ot nun sulunc clergie,

Muement de rei senefie.

Clere esteit merveillusement,

Si getot un rai sulement.

Uns feus, ki de cel rai eisseit,

Figure de dragun faiseit ;

De cel dragun dui rai veneient $[. . .]^{58}$

Ce phénomène, perçu comme un "signe " par le peuple, et dont Uter se "merveilla", appelle des explications. Merlin est donc prié de dire «que si fait signe senefie ». C'est alors que le prophète révèle la mort d'Aurèle puis réconforte Uter en lui annonçant la prospérité de ses enfants futurs. Le dragon symbolisait Uter lui-même, et les rayons, ses descendants. 
61 Robert de Boron conserve et développe ce passage du Brut. Dédaignant le mot «cumete », il donne à l'événement une belle ampleur, le faisant d'abord annoncer par la bouche de Merlin :

[...] vos verrez .I. dragon vermeil qui corra par l'air entre le ciel et la terre. ${ }^{59}$ Le prodige advient comme annoncé, et par la suite, Merlin donnera la «senefiance dou dragon", en la limitant seulement à Uter, et en lui demandant d'adopter en conséquence le nom d'Uitierpandragon. Toute allusion à la postérité est supprimée, la merveille brille isolément dans sa splendeur signifiante.

On touche là à une dernière modification d'importance. Dans le Brut, l'absence des vaticinations un peu délirantes que l'on trouve dans l'Historia Regum Britanniae maintient Merlin dans un rôle de prophète bienséant, dont la fonction s'apparente à celle des devins antiques ou bibliques, tels qu'il s'en rencontre à l'occasion dans le Brut lui-même ${ }^{60}$. Surtout, ses prédictions, peu nombreuses, renvoient au devenir imminent du récit en annonçant les règnes successifs d'Aurèle, Uter et Arthur; en somme, elles renforcent l'axe chronologique, elles sont un auxiliaire de la structuration temporelle de l'œuvre ${ }^{61}$. Dans la prose, par contre, la lecture de l'avenir reçoit des illustrations plaisantes et en partie gratuites : je citerai simplement l'annonce d'un décès par noyade et pendaison simultanée. La fantaisie de Merlin est également illustrée par son goût pour les métamorphoses, ce dont on ne trouve aucun équivalent chez Wace.

Merlin devient donc dans la prose un être merveilleux. Son caractère surnaturel se manifeste nettement à l'occasion du transport des pierres de Stonehenge d'Irlande en Grande-Bretagne, opération sur laquelle Wace reste très concis, présentant la chose de telle sorte que l'on peut estimer que c'est l'ingenium du prophète qui a rendu possible le transfert ${ }^{62}$. Au contraire, dans le Merlin, la «merveille des pierres » est complaisamment soulignée, au moment où le peuple se rend à Salesbières pour voir le prodige :

Quant il furent la et il les virent, si distrent que touz li mondes n'en deust pas une lever ne que tiels pierres n'ossast en pas mestre sor mer en vaissiaus. Molt se merveillent coment il les avoit faites venir, que nus ne l'avoit veu ne seu. ${ }^{63}$

En un endroit seulement, Wace échoue à rationaliser l'activité de Merlin, lorsque ce dernier change sa propre physionomie ainsi que celles d'Uter et d'Ulfin afin d'entrer dans Tintagel. Wace utilise alors le mot qui s'impose, "enchantemenz " ${ }^{64}$. La présence $\mathrm{du}$ fils de diable finit par introduire de l'irrationnel dans le récit! Mais soixante vers plus loin, dès que chacun des personnages a retrouvé son visage, l'enchanteur disparaît définitivement de la diégèse ${ }^{65}$. Il en va tout autrement dans le Merlin, où l'épisode des métamorphoses se voit au contraire développé.

En somme, le Merlin s'approprie la part la moins historique - et la plus fictionnelle - du Brut et la développe, au mépris absolu de la véridicité, dont son prédécesseur se montrait pourtant fort soucieux. On comprend alors la pertinence du métalangage employé dans les deux œuvres, les raisons qui justifient la présence d' «estorie» ou " geste » dans le Brut, leur absence dans le Merlin, où l'on trouve « conte » et « livre ».

Cette confrontation permet de la sorte de reconsidérer quelques idées reçues, en particulier les liens, que l'on dit forts, entre la prose et le temps d'une part, la prose et la véridicité d'autre part. La prose du Merlin se met au service de ce qui, dans l'historiographie, s'oriente vers le domaine de la fiction, comme si l'écrivain qui apparaît sous le nom de Robert de Boron s'était attaché à utiliser tout particulièrement les données déviantes du Brut et à développer les traits marginaux de son modèle. C'est 
la logique du personnage principal qu'il adopte et qu'il approfondit, en créant la mise en scène d'une autobiographie. La hiérarchisation générique du Brut, remarquable par sa stabilité, laisse alors place à une hybridation évolutive dans le Merlin, où le modèle de Wace fournit cependant un cadre événementiel et l'aspiration à une continuité de la diégèse. Le fils du diable est ainsi l'artisan d'un récit au genre énigmatique, et le responsable d'une association entre prose et fictionnalité que développeront par la suite les grands romans chevaleresques.

\section{NOTES}

1.Le texte en vers a été publié sous le titre Le Roman de l'Estoire dou Graal de Robert de Boron, éd. par William A. Nitze, Paris, Champion (CFMA 57), 1927. Le texte en prose est accessible dans Le Roman du Graal, manuscrit de Modène, texte établi et présenté par Bernard Cerquiglini, Paris, UGE 10/18 (Bibliothèque médiévale), 1981 ; cet ouvrage contient également l'ensemble du premier cycle en prose, que l'on appelle parfois le Petit Saint Graal, et qui est formé d'une suite de trois récits : le Joseph, le Merlin et le Perceval. Il existe maintenant une édition qui met en parallèle les Joseph en vers et en prose : Robert de Boron, Joseph d'Arimathie, éd. par Richard O'Gorman, Toronto, Pontifical Institute of Mediaeval Studies (Studies and Textes 120), 1995.

2.Cf., sur ce sujet, Bernard Cerquiglini, «Sur la prose de Joseph d'Arimathie. Forme et statut de la parole », Perspectives médiévales, 3, oct. 1977, pp. 43-49 et discussion pp. 52-56 ; La Parole médiévale. Discours, syntaxe, texte, Paris, Minuit, 1981. Et aussi, Richard F. O'Gorman, «The Prose Version of Robert de Boron's Joseph d'Arimathie », Romance Philology, 23, 1970, pp. 449-61.

3.Robert de Boron, Merlin. Roman du XIII e siècle, éd. par Alexandre Micha, Genève, Droz (TLF 281), 1980. Sur le Merlin, il existe deux études de référence : Paul Zumthor, Merlin le prophète. Un thème de la littérature polémique, de l'historiographie et des romans, Lausanne, Payot, 1943 (réimpr. Genève, Slaktine, 1973); Alexandre Micha, Etude sur le « Merlin » de Robert de Boron, roman du XIII siècle, Genève, Droz (Publications Romanes et Françaises CLI), 1980.

4.C'est dans l'unique manuscrit comportant le Joseph en vers que figurent ces cinq cents vers. On les trouve publiés dans le Roman de l'Estoire dou Graal, éd. par Nitze (op. cit., pp. 126-30), ainsi que dans le Merlin éd. par Micha (op. cit., pp. 1-17).

5.Sur cette tradition manuscrite, cf. Alexandre Micha, « Les manuscrits du Merlin en prose de Robert de Boron ", Romania, 78, 1957, pp. 78-94 et pp. 145-74.

6.Wace, Le Roman de Brut, éd. par Ivor Arnold, 2 vol., 1938-40, Firmin-Didot, Paris, (SATF). La partie arthurienne du Brut et de l'Historia Regum Britanniae de Geoffroy de Monmouth sont accessibles dans La Geste du roi Arthur selon le «Roman de Brut » de Wace et l'« Historia Regum Britanniae » de Geoffroy de Monmouth, présentation, édition et traductions par E. Baumgartner et I. Short, Paris, U. G. E., 10/18 (Bibliothèque Médiévale), 1993.

Alexandre Micha a précisément étudié la dette de Robert de Boron au niveau événementiel. Cf. son Etude sur le « Merlin »..., op. cit., pp. 30-37. L'auteur s'interroge sur 
les rapports éventuels entre le Merlin et l'Historia Regum Britanniae de Geoffroy de Monmouth. Il constate quelques cas de proximité, «mais ces quelques rencontres, possibles souvenirs épars d'une lecture de l'Historia, sont infiniment plus rares que les précédentes [avec le Brut], et c'est bien un Brut que Robert de Boron a eu sous les yeux » (p. 37).

7.Merlin., op. cit. p. 75.

8.Ma réflexion s'inspire indirectement du travail de Jean-Marie Schaeffer dans son ouvrage Qu'est-ce qu'un genre littéraire ?, Paris, Seuil (Poétique), 1989, mais sans en reprendre toutes les catégories, qui conviennent davantage à la littérature moderne.que médiévale. Je lui emprunte ici, en l'adaptant, la notion de «trait générique ».

9.Le mot « récit » sera compris de façon minimale comme se rapportant à des textes qui présentent une dimension narrative manifeste. C'est un trait que partagent tous les écrits attribués à Robert de Boron, comme aussi ceux de Wace ou de Chrétien de Troyes. 10.Merlin, op. cit., p. 76 et p. 290. Les deux passages en question ne figurent pas dans tous les manuscrits.

11.Les deux premières citations sont extraites de la page 82 (ibid.), la troisième des pp. 138-39. Cf., également, « Merlin li conta les choses, et Blaises le mist en son livre et par son livre le resavons encor » (p. 121).

12.Ibid., p. 190.

13.Ibid, respectivement, p. 73, p. 74, p. 235 et p. 163. Cet échantillon aurait pu être étendu.

14.Ibid., p. 76 et p. 85. Merlin emploie aussi le verbe « retraire », p. 75 .

15. En deux occasions on trouve un autre déterminant que le démonstratif: p. 82, «li » et p. 198, «mes ». Robert de Boron ne s'approprie le mot « livre » qu'à la fin du Merlin : «Et je Rebert de Borron qui cest livre retrais par l'enseingnement dou Livre dou Graal... ». Cet emploi tardif du mot « livre » est certainement rendu possible parce que l'œuvre « retraite » est maintenant terminée.

16.Ibid., p. 175.

17. Cette scène rappelle l'échange verbal entre Satan et Enfer que l'on peut lire dans l'Évangile de Nicodème. Cf. Trois versions rimées de l'Évangile de Nicodème par Chrétien, André de Coutances et un anonyme, publiées par Gaston Paris et Alphonse Bos, Paris, FirminDidot, 1885.

18.Merlin, op. cit., p. 23, p. 26 et p. 27.

19.Le « miraculeux » renvoie à la tradition chrétienne, alors que le « merveilleux » concerne le domaine légendaire, celtique notamment. Mais parfois les deux traits se rejoignent, par exemple au moment de l'élection d'Arthur.

20. Comme l'écrit Alexandre Micha, « la distorsion chronologique s'explique par l'existence de deux chaînes temporelles, l'une relevant du temps mythique, l'autre du temps humain. » (Etude sur le « Merlin »..., op. cit., p. 153).

21.Merlin, op. cit, p. 76.

22.Etude sur le « Merlin »..., op. cit., p. 150.

23.C'est-à-dire l'élaboration du Merlin et du Joseph, puisque Merlin dicte à Blaise le contenu de ce livre-là, mentionné sous forme de résumé pp. 73-74 (ibid.).

24.Cf., dans le ms BN f. fr. 794, la variante des vv. 7-8 que cite l'éditeur : « Cil reconte la verité/Qui lo latin a translaté ». 
25.Robert Marichal, " Naissance du roman ", Entretiens sur la Renaissance du XII ${ }^{e}$ siècle, sous la direction de Maurice de Gandillac et Édouard Jeauneau, Paris, Mouton, 1968, pp. 449-82 et discussion pp. 483-92.

26.Brut, op. cit., vv. 103-104.

27.Ainsi au v. 940 (op. cit.) : « si com nus leü avum » et juste après, à propos d'une explication qui va être donnée : « si come jo la truis escrite. » (v. 946).

28.Ibid., v. 3823 et v. 14866 .

29.Ibid., vv. 13282-84.

30.Ibid., vv. 10359-61. Cf. également, référant de la même façon au livre-source :

«Arthur, si la geste ne ment, / Fud el cors nafrez mortelment ; », vv. 13275-76. Et pour le mot « estorie » employé seul, les vv. 1597 (« ço dist l'estorie ») et 2310 (« Si come l'estorie recorde »).

31.Ibid., v. 14859.

32.Geffrei Gaimar, Estoire des Engleis, éd. par Alexander Bell, Oxford, Blackwell (AngloNorman Text Society), 1960. Gaimar, s'il avait écrit en anglais, aurait pu écrire en prose, car il existait déjà une tradition de prose littéraire dans cette langue. Mais en français, il n'avait pas le choix, comme l'explique Alexander Bell dans son article « Gaimar as pioneer ", Romania, 97, 1976, p. 465. Les modèles existants vers 1140 étaient le Voyage de Saint Brendan et, en partie, le Bestiaire et les Lapidaires de Philippe de Thaon.

33.Brut, op. cit., v. 5.

34.Ibid., respectivement v. 21, v. 24, v. 1455 (le vers 2307 est identique).

35.Brut., op. cit., vv. 1023-26. Les exemples sont innombrables. Il est ainsi question de Bretainne (v. 1175sq.), Cornervaille (v. 1186sq.), Lundres (par la filiation Troie Nove, " puis ala li nuns corumpant ", Trinovant et, après le règne de Lud, Kaerlu, altéré en Lodoïn, v. 1221sq.).

36.Ibid., vv. 7-8.

37.Ibid., vv. 3821-23.

38.Les exemples sont très nombreux. Voici par exemple ce qu'écrit Wace du château des Pucelles, dont il ne sait pas justifier le nom : « Mais jo ne sai par quel raisun/Li chastels out nun des Pulceles/Plus que dames ne d'anceles;/Ne me fu dit ne jo nel di/ $\mathrm{Ne}$ jo n'ai mie tut oï/Ne jo n'ai mie tut veü/Ne jo n'ai pas tut entendu,/E mult estovreit home entendre/Ki de tut vuldreit raison rendre ». (Ibid., vv. 1528-31). On pourrait aussi mentionner ce commentaire introduit à propos des géants qui peuplaient l'Angleterre : « Ne vus sai lur nuns aconter/Ne nul n'en sai, fors un, nomer./L'un sai nomer, cel vus puis dire, / Goëmagog, qui ert lur sire. » (vv. 1067-70).

39.Ibid., vv. 7539-42: « Ne vuil sun livre translater/Quant jo nel sai interpreter ;/Nule rien dire ne vuldreie/Que si ne fust cum jo dirreie ». Ces prophéties figurent dans l'œuvre de Geoffroy de Monmouth. Cf. The Historia regum Britanniae of Geoffrey of Monmouth, I, éd. par Neil Wright, Cambridge, D. S. Brewer, 1985, §§ 111-17.

40.Ibid., vv. 8659-65.

41.Ibid., vv. 1111-68.

42.Ibid., vv. 1117-26.

43. Toutefois, une tonalité épique pointe parfois lorsque des combats sont racontés brièvement et plus sobrement, et que la férocité des guerriers est soulignée. $\mathrm{Cf}$. le passage où les Troyens affrontent les Grecs (Ibid., v. 295sq). Cf., également, v. 7753sq. 44. Historia Regum Britanniae, op. cit., § 17. Voici la traduction que L. Mathey-Maille donne de cette phrase : « Là leur apparurent les monstres marins appelés Sirènes : elles entourèrent les navires et peu s'en fallut qu'elles ne les fissent sombrer. » (Geoffroy de 
Monmouth, Histoire des rois de Bretagne, Paris, Les Belles Lettres (La roue à livres), 1993, p. 42). Dans le Brut (op. cit.), vv. 733-64.

45.Brut, op. cit., vv. 637-38.

46.Cf. les analyses d'Alexandre Micha, Etude sur le « Merlin »..., op. cit., pp. 37-48. Par exemple, l'épisode de Stonehenge se situe avant la mort du frère d'Uter dans le Brut, et seulement après dans le Merlin.

47.Ibid., v. 1247. C'est la première allusion du Brut au temps biblique.

48.Ibid., respectivement vv. 1248, 1469, 4874. La liste n'est pas exhaustive !

49.Ibid., vv. 8407-8540. Pour plus de détails sur ces élagages, je renvoie aux pages

d'Alexandre Micha, Etude sur le « Merlin »..., op. cit., pp. 37-48.

50.Merlin, op. cit., p. 76 et p. 85.

51.Merlin le prophète..., op. cit., p. 167.

52.Il faut aussi mentionner le cas unique où l'on peut trouver une transition qui évoque le procédé de l'entrelacement : «De ces .II. enfanz ne doi je plus parler tant que li contes m'i ramaint. » (Ibid., p. 82). Mais cette phrase souligne davantage un changement d'acteur qu'une rupture temporelle.

53.Ibid., p. 43.

54.Ibid., p. 78. Cf., également, « Einsis remest une grant piece tant que il vint au setenne jor devant que elle devoit estre arse » (p. 56). Les exemples sont nombreux de constructions de ce type. Le verbe « remestre " peut à l'occasion favoriser un changement d'acteur, comme le feront plus tard, dans le Lancelot, les formules de transition " Mais or se taist li contes de... et parole de... ». Ainsi, p. 163, on trouve l'enchaînement « Einsi remest dou roi. Merlins s'en ala en Northumbellande... ». 55.Ibid., v. 7431.

56.Ibid., vv. 7439-41 et v. 7445 .

57.La démarche est la même dans l'Historia Regum Britanniae. Le clerc, dont est donné le nom (Maugantius) explique : " J'ai lu dans les livres de nos philosophes et dans de nombreuses histoires que beaucoup d'hommes ont été conçus de la sorte. Car ainsi que le raconte Apulée à propos du dieu de Socrate, des esprits que nous appelons démons incubes habitent entre la lune et la terre [...] ». On voit que Wace est ici extrêmement près de son modèle. § 107, traduction de L. Mathey-Maille, op. cit., p. 155.

58.Brut, op. cit., vv. 8289-95.

59.Merlin, op. cit., p. 172.

60.Ainsi du dénommé Teleusin, qualifié de devin et de bon prophète, qui surgit brièvement dans le récit pour annoncer la naissance du Christ (Brut, op. cit., vv. 4855-76). Sa prédiction, inutile de le préciser, s'avère fondée. Teleusin, qui précède Merlin dans le déroulement du Brut, prouve la respectabilité des prophètes lorsqu'ils ont affaire aux choses de la chrétienté.

61.Elles adviennent deux fois. D'abord, Vertigier demande à Merlin, qu'il tient « a buen devin ", de lui dévoiler la date et les circonstances de sa mort. Merlin lui annonce une fin prochaine, puis nomme les rois qui lui succéderont : Aurèle, Uter et le fils de ce dernier (Ibid., vv. 7548-82). Le second discours prophétique, adressé à Uter, évoque les futurs enfants du roi, dont un fils « ki conquerra jesqu'ultre France » (vv. 8325-44). 62.Ce qui concerne la carole des géants couvre les vers 8119-78 du Brut (op. cit.). Auparavant, Merlin a expliqué au roi que « engin surmunte vertu./Bone est force e engin mielz valt ; (vv. 8059-60).

63.Merlin, op. cit., p. 181.

64.Brut., op. cit., v. 8727 : «Merlin fist ses enchantemenz,/Vis lur mua et vestemenz ». 
65.Merlin quitte donc le récit sans cérémonie : «Quant lors furent en lur chemin/Li reis e Ulfin e Merlin,/Tels fu chescun cum estre dut/E chescuns sa furme reçut. » (Ibid., vv. 8787-90). On ne sait nullement ce que va devenir le prophète...

\section{AUTEUR}

\section{ANNIE COMBES}

Université de Nantes 